\title{
DESIGN AND ANALYSIS OF A SHOCK ABSORBER
}

\author{
Pinjarla.Poornamohan ${ }^{1}$, Lakshmana Kishore. $\mathbf{T}^{2}$ \\ ${ }^{1}$ M.Tech (student), ${ }^{2}$ M.Tech (Associate Professor), Mechanical Department, GIET, Andhra Pradesh, India \\ poornamohan_mech@yahoo.com, tlkishore.giet@gmail.com
}

\begin{abstract}
A suspension system or shock absorber is a mechanical device designed to smooth out or damp shock impulse, and dissipate kinetic energy. The shock absorbers duty is to absorb or dissipate energy. In a vehicle, it reduces the effect of traveling over rough ground, leading to improved ride quality, and increase in comfort due to substantially reduced amplitude of disturbances. When a vehicle is traveling on a level road and the wheels strike a bump, the spring is compressed quickly. The compressed spring will attempt to return to its normal loaded length and, in so doing, will rebound past its normal height, causing the body to be lifted. The weight of the vehicle will then push the spring down below its normal loaded height. This, in turn, causes the spring to rebound again. This bouncing process is repeated over and over, a little less each time, until the up-and-down movement finally stops. If bouncing is allowed to go uncontrolled, it will not only cause an uncomfortable ride but will make handling of the vehicle very difficult. The design of spring in suspension system is very important. In this project a shock absorber is designed and a 3D model is created using Pro/Engineer. The model is also changed by changing the thickness of the spring. Structural analysis and modal analysis are done on the shock absorber by varying material for spring, Spring Steel and Beryllium Copper. The analysis is done by considering loads, bike weight, single person and 2 persons. Structural analysis is done to validate the strength and modal analysis is done to determine the displacements for different frequencies for number of modes. Comparison is done for two materials to verify best material for spring in Shock absorber. Modeling is done in Pro/ENGINEER and analysis is done in ANSYS. Pro/ENGINEER is the standard in $3 D$ product design, featuring industry-leading productivity tools that promote best practices in design.ANSYS is general-purpose finite element analysis (FEA) software package. Finite Element Analysis is a numerical method of deconstructing a complex system into very small pieces (of user-designated size) called elements.
\end{abstract}

Index Terms: damp shock, kinetic energy, Pro/Engineer, and ANSYS, shock absorber

\section{INRODUCTION}

A shock absorber or damper is a mechanical device designed to smooth out or damp shock impulse, and dissipate kinetic energy.

\subsection{Description}

Pneumatic and hydraulic shock absorbers commonly take the form of a cylinder with a sliding piston inside. The cylinder is filled with a fluid (such as hydraulic fluid) or air. This fluidfilled piston/cylinder combination is a dashpot.

\subsection{Explanation}

The shock absorbers duty is to absorb or dissipate energy. One design consideration, when designing or choosing a shock absorber, is where that energy will go. In most dashpots, energy is converted to heat inside the viscous fluid. In hydraulic cylinders, the hydraulic fluid will heat up, while in air cylinders, the hot air is usually exhausted to the atmosphere. In other types of dashpots, such as electromagnetic ones, the dissipated energy can be stored and used later. In general terms, shock absorbers help cushion cars on uneven roads.

\subsection{Applications}

Shock absorbers are an important part of automobile and motorcycle suspensions, aircraft landing gear, and the supports for many industrial machines. Large shock absorbers have also been used in structural engineering to reduce the susceptibility of structures to earthquake damage and resonance. A transverse mounted shock absorber, called a yaw damper, helps keep railcars from swaying excessively from side to side and are important in passenger railroads, commuter rail and rapid transit systems because they prevent railcars from damaging station platforms. The success of passive damping technologies in suppressing vibration amplitudes could be ascertained with the fact that it has a market size of around \$ 4.5 billion.

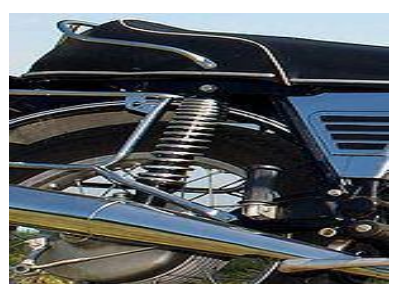

Fig 1.1:- Rear shock absorber and spring of a BMW R75/5 motorcycle 


\subsection{Vehicle suspension}

In a vehicle, it reduces the effect of traveling over rough ground, leading to improved ride quality, and increase in comfort due to substantially reduced amplitude of disturbances. Without shock absorbers, the vehicle would have a bouncing ride, as energy is stored in the spring and then released to the vehicle, possibly exceeding the allowed range of suspension movement. Control of excessive suspension movement without shock absorption requires stiffer (higher rate) springs, which would in turn give a harsh ride. Shock absorbers allow the use of soft (lower rate) springs while controlling the rate of suspension movement in response to bumps. They also, along with hysteresis in the tire itself, damp the motion of the unspring weight up and down on the springiness of the tire. Since the tire is not as soft as the springs, effective wheel bounce damping may require stiffer shocks than would be ideal for the vehicle motion alone. Spring-based shock absorbers commonly use coil springs or leaf springs, though torsion bars can be used in tensional shocks as well. Ideal springs alone, however, are not shock absorbers as springs only store and do not dissipate or absorb energy. Vehicles typically employ springs and torsion bars as well as hydraulic shock absorbers. In this combination, "shock absorber" is reserved specifically for the hydraulic piston that absorbs and dissipates vibration.

\subsection{Structures}

Applied to a structure such as a building or bridge it may be part of a seismic retrofit or as part of new, earthquake resistant construction. In this application it allows yet restrains motion and absorbs resonant energy, which can cause excessive motion and eventual structural failure.

\subsection{Types of shock absorbers}

There are several commonly-used approaches to shock absorption:

- Hysteresis of structural material, for example the compression of rubber disks, stretching of rubber bands and cords, bending of steelsprings, or twisting of torsion bars. Hysteresis is the tendency for otherwise elastic materials to rebound with less force than was required to deform them. Simple vehicles with no separate shock absorbers are damped, to some extent, by the hysteresis of their springs and frames.

- Dry friction as used in wheel brakes, by using disks (classically made of leather) at the pivot of a lever, with friction forced by springs. Used in early automobiles such as the Ford Model T, up through some British cars of the 1940s. Although now considered obsolete, an advantage of this system is its mechanical simplicity; the degree of damping can be easily adjusted by tightening or loosening the screw clamping the disks, and it can be easily rebuilt with simple hand tools. A disadvantage is that the damping force tends not to increase with the speed of the vertical motion.

- Solid state, tapered chain shock absorbers, using one or more tapered, axial alignment(s) of granular spheres, typically made of metals such as nitinol, in a casing. [1],[2]

- Fluid friction, for example the flow of fluid through a narrow orifice (hydraulics), constitutes the vast majority of automotive shock absorbers. An advantage of this type is that using special internal valving the absorber may be made relatively soft to compression (allowing a soft response to a bump) and relatively stiff to extension, controlling "jounce", which is the vehicle response to energy stored in the springs; similarly, a series of valves controlled by springs can change the degree of stiffness according to the velocity of the impact or rebound. Specialized shock absorbers for racing purposes may allow the front end of a dragster to rise with minimal resistance under acceleration, then strongly resist letting it settle, thereby maintaining a desirable rearward weight distribution for enhanced traction. Some shock absorbers allow tuning of the ride via control of the valve by a manual adjustment provided at the shock absorber. In more expensive vehicles the valves may be remotely adjustable, offering the driver control of the ride at will while the vehicle is operated. The ultimate control is provided by dynamic valve control via computer in response to sensors, giving both a smooth ride and a firm suspension when needed. Many shock absorbers contain compressed nitrogen, to reduce the tendency for the oil to foam under heavy use. Foaming temporarily reduces the damping ability of the unit. In very heavy duty units used for racing and/or offroad use, there may even be a secondary cylinder connected to the shock absorber to act as a reservoir for the oil and pressurized gas. Another variation is the Magneto rheological damper which changes its fluid characteristics through an electromagnet.

- Compression of a gas, for example pneumatic shock absorbers, which can act like springs as the air pressure is building to resist the force on it. Once the air pressure reaches the necessary maximum, air dashpots will act like hydraulic dashpots. In aircraft landing gear air dashpots may be combined with hydraulic damping to reduce bounce. Such struts are called oleo struts (combining oil and air) [3].

- Magnetic effects. Eddy current dampers are dashpots that are constructed out of a large magnet inside of a non-magnetic, electrically conductive tube.

- Inertial resistance to acceleration, for example prior to 1966 [4] the Citroën 2CV had shock absorbers that damp wheel bounce with no external moving parts. These consisted of a spring-mounted $3.5 \mathrm{~kg}(7.75 \mathrm{lb})$ 
iron weight inside a vertical cylinder [5] and are similar to, yet much smaller than versions of the tuned mass dampers used on tall buildings

- Composite hydro-pneumatic devices which combine in a single device spring action, shock absorption, and often also ride-height control, as in some models of the Citroën automobile.

- Conventional shock absorbers combined with composite pneumatic springs with which allow ride height adjustment or even ride height control, seen in some large trucks and luxury sedans such as certain Lincoln and most Land Rover automobiles. Ride height control is especially desirable in highway vehicles intended for occasional rough road use, as a means of improving handling and reducing aerodynamic drag by lowering the vehicle when operating on improved high speed roads.

- the effect of a shock absorber at high (sound) frequencies is usually limited by using a compressible gas as the working fluid and/or mounting it with rubber bushings.

- The detailed analysis of shock absorber/isolation systems is very complicated and involves assessment of the dynamic response of the equipment to different types of activating energy inputs. The notes below relate only to illustrating the benefits of using shock absorbers to reduce the forces experienced by equipment to impacts. The more complicated scenarios involving systems continuously operating and withstanding sudden changes of loading and acceleration e.g., car suspension systems and aircraft landing gear, are outside of the scope of this work. Moving objects have kinetic energy related to their velocity and their mass. If the velocity of an object is significantly changed in a short time span e.g. it impacts on a stationary body, then high forces result. These forces can be useful e.g., a forging press using the kinetic energy to form metal. However real life impact forces (shock loads) are generally very destructive and are avoided. Kinetic energy increases in a direct ratio to the mass and to the velocity squared.

- The heavier the object or the faster it travels, the more energy it has. Methods of energy absorption include rubber buffers, metal springs, air springs, and hydraulic shock absorbers. When the systems have to continuously operate under the influence of shock loads the shock isolation system generally includes spring-dashpot isolation systems. For simple shock absorber applications required to mitigate the effect of a single events then viscous dampers which dissipate the energy, as heat rise of a fluid, are often preferred. In normal everyday life simple examples of shock absorber systems include crash helmets, steel toe caps in industrial boots, collapsible bumpers on cars, motor way barriers. The notes below are general in nature provided to show the benefits of using shock absorbers. For more detailed information - links are provided to shock absorber suppliers.

\subsection{Shock Absorber types}

There are a number of different methods of converting an impact /collision into relatively smooth cushioned contact..

- Metal Spring

- Rubber Buffer

- Hydraulic Dashpot

- Collapsing safety Shock Absorbers

- Pneumatic Cylinders

- Self compensating Hydraulic

\subsubsection{Metal springs}

Simply locating metal springs to absorb the impact loads are a low cost method of reducing the collision speed and reducing the shock loading. They are able to operate in very arduous conditions under a wide range of temperatures. These devices have high stopping forces at end of stroke. Metal springs store energy rather than dissipating it. If metal sprint type shock absorbers are used then measures should be provided to limit Oscillations. Metal springs are often used with viscous dampers.

There are a number of different types of metal springs including helical springs, bevel washers(cone-springs), leaf springs, ring springs, mesh springs etc etc. Each spring type has its own operating characteristics.

\subsubsection{Elastomatic shock observers}

These are low cost options for reducing the collision speed and reducing the shock loading and providing system damping. They are conveniently moulded to suitable shapes. These devices have high stopping forces at end of stroke with significant internal damping. Elastomeric dampers are very widely used because of the associated advantages of low cost and mouldability together with performance benefits. The inherent damping of elastomers is useful in preventing excessive vibration amplitude at resonance - much reduced compared to metal springs. However elastomeric based shock absorbers are limited in being affected by high and low temperatures. And are subject to chemical attack. Silicone rubber is able to provide reasonable mechanical properties between temperatures of -500 to $+180 \mathrm{O}$ deg. C- most other elastomer has inferior temperature tolerance.

\subsubsection{Hydraulic Dashpot}

This type of shock absorber is based on a simple hydraulic cylinder. As the piston rod is moved hydraulic fluid is forced through an orifice which restricts flow and consequently provides a controlled resistance to movement of the piston rod. With only one metering orifice the moving load is abruptly slowed down at the start of the stroke. The braking 
force rises to a very high peak at the start of the stroke and then falls away rapidly. On completion of the stroke the system is stable - the energy being dissipated in the hydraulic fluid as heat. This type of shock absorbers are provided with Springs sufficient to return the actuator to its initial position after the impacting load is removed.

\subsubsection{Collapsing Safety Shock Absorbers}

These are single use units which are generally specially designed for specific duties. They are designed such that at impact they collapse and the impact energy is absorbed as the materials distort in their inelastic/yield range. They therefore are more compact compared to devices based on deflections within their elastic range.

\subsubsection{Air (Pneumatic) spring}

These devices use air as the resilient medium. Air has a high energy storage capacity compared to metal or elastomer materials. For duties with high loads and deflections the air spring is generally far more compact that the equivalent metal or elastomer device. Due to the compressibility of air these have a sharply rising force characteristic towards the end of the stroke. The majority of the energy is absorbed near the end of the stroke.The force on an air cylinder buffer is determined by the relation $\mathrm{PVn}=$ constant. Air springs require more maintenance than meal or elastomer based springs and the temperature range is restricted compared to metal springs.

\subsubsection{Self compensating Hydraulic}

These devices are similar to the hydraulic dashpot type except that a number of orifices are provided allowing different degrees of restriction throughout the stroke. These devices are engineered to bring the moving load is smoothly and gently to rest by a constant resisting force throughout the entire shock absorber stroke. The load is decelerated with the lowest possible force in the shortest possible time eliminating damaging force peaks and shock damage to machines and equipment. These type of shock absorbers are provided with springs sufficient to return the actuator to its initial position after the impacting load is removed.

\subsection{Design Calculations for Helical springs for Shock}

\section{absorbers}

Material: Steel(modulus of rigidity) $G=41000$

Mean diameter of a coil $\mathrm{D}=62 \mathrm{~mm}$

Diameter of wire $\mathrm{d}=8 \mathrm{~mm}$

Total no of coils $n 1=18$

Height $\mathrm{h}=220 \mathrm{~mm}$

Outer diameter of spring coil $\mathrm{D} 0=\mathrm{D}+\mathrm{d}=70 \mathrm{~mm}$

No of active turns $n=14$

Weight of bike $=125 \mathrm{kgs}$

Let weight of 1 person $=75 \mathrm{Kgs}$

Weight of 2 persons $=75 \times 2=150 \mathrm{Kgs}$
Weight of bike + persons $=275 \mathrm{Kgs}$

Rear suspension $=65 \%$

$65 \%$ of $275=165 \mathrm{Kgs}$

Considering dynamic loads it will be double

$\mathrm{W}=330 \mathrm{Kgs}=3234 \mathrm{~N}$

For single shock absorber weight $=\mathrm{w} / 2=1617 \mathrm{~N}=\mathrm{W}$

We Know that, compression of spring $(\delta)=\times \times / \times$

$\mathrm{C}=$ spring index $===7.75=8$

$(\delta)=\times \times \times / \times=282.698$

Solid length, $\mathrm{Ls}=\mathrm{n} 1 \times \mathrm{d}=18 \times 8=144$

Free length of spring,

$\mathrm{Lf}=$ solid length + maximum compression + clearance between adjustable coils

$=++0.15=144+282.698+0.15 \times 282.698=469.102$

Spring rate, $\mathrm{K}==.=5.719$

Pitch of coil, $\mathrm{P}==.=26$

Stresses in helical springs: maximum shear stress induced in the wire

$\tau=\mathrm{K} \times$

$\mathrm{K}=+.=\times$

$\mathrm{x}+.=0.97$

$\tau=\mathrm{K} \times 0.97 \times \times \times$

$x=499.519$

Buckling of compression springs, $=\times \times$

Values of buckling factor $\mathrm{KB}=7.5$

$\mathrm{K}=0.05$ (for hinged and spring)

The buckling factor for the hinged end and built-in end springs $\mathrm{Wcr}=5.719 \times 0.05 \times 469.102=134.139 \mathrm{~N}$

\section{INTRODUCTION TO PRO/ENGINEER}

Pro/ENGINEER is a feature based, parametric solid modeling program. As such, it's use is significantly different from conventional drafting programs. In conventional drafting (either manual or computer assisted), various views of a part are created in an attempt to describe the geometry. Each view incorporates aspects of various features (surfaces, cuts, radii, holes, protrusions) but the features are not individually defined. In feature based modeling, each feature is individually described then integrated into the part. The other significant aspect of conventional drafting is that the part geometry is defined by the drawing. If it is desired to change the size, shape, or location of a feature, the physical lines on the drawing must be changed (in each affected view) then associated dimensions are updated. When using parametric modeling, the features are driven by the dimensions (parameters). To modify the diameter of a hole, the hole diameter parameter value is changed. This automatically modifies the feature wherever it occurs - drawing views, assemblies, etc. Another unique attribute of Pro/ENGINEER is that it is a solid modeling program. The design procedure is to create a model, view it, assemble parts as required, then generate any drawings which are required. It should be noted that for many uses of Pro/E, complete drawings are never created. A typical design cycle for a molded plastic part might consist of the creation of a solid model, export of an SLA file to a rapid prototyping system (stereolithography, etc.), use of 
the SLA part in hands-on verification of fit, form, and function, and then export of an IGES file to the molder or toolmaker. A toolmaker will then use the IGES file to program the NC machines which will directly create the mold for the parts. In many such design cycles, the only print created will be an inspection drawing with critical and envelope dimensions shown.

\subsection{Summary of capabilities}

Like any software it is continually being developed to include new functionality. The details below aim to outline the scope of capabilities to give an overview rather than giving specific details on the individual functionality of the product. Pro/Engineer is a software application within the CAID/CAD/CAM/CAE category, along with other similar products currently on the market. Pro/Engineer is a parametric, feature-based modeling architecture incorporated into a single database philosophy with advanced rule-based design capabilities. The capabilities of the product can be split into the three main heading of Engineering Design, Analysis and Manufacturing. This data is then documented in a standard 2D production drawing or the 3D drawing standard ASME Y14.41-2003.

\subsection{Engineering Design}

Pro/Engineer offers a range of tools to enable the generation of a complete digital representation of the product being designed. In addition to the general geometry tools there is also the ability to generate geometry of other integrated design disciplines such as industrial and standard pipe work and complete wiring definitions. Tools are also available to support collaborative development. A number of concept design tools that provide up-front Industrial Design concepts can then be used in the downstream process of engineering the product. These range from conceptual Industrial design sketches, reverse engineering with point cloud data and comprehensive freeform surface tools.

\subsection{DIFFERENT MODULES IN PRO/ENGINEER}

- PART DESIGN

- ASSEMBLY

- DRAWING

- SHEETMETAL

\section{MODEL OF SHOCK ABSORBER}

\subsection{PARTS OF SHOCK ABSORBER}

\subsubsection{BOTTOM PART}

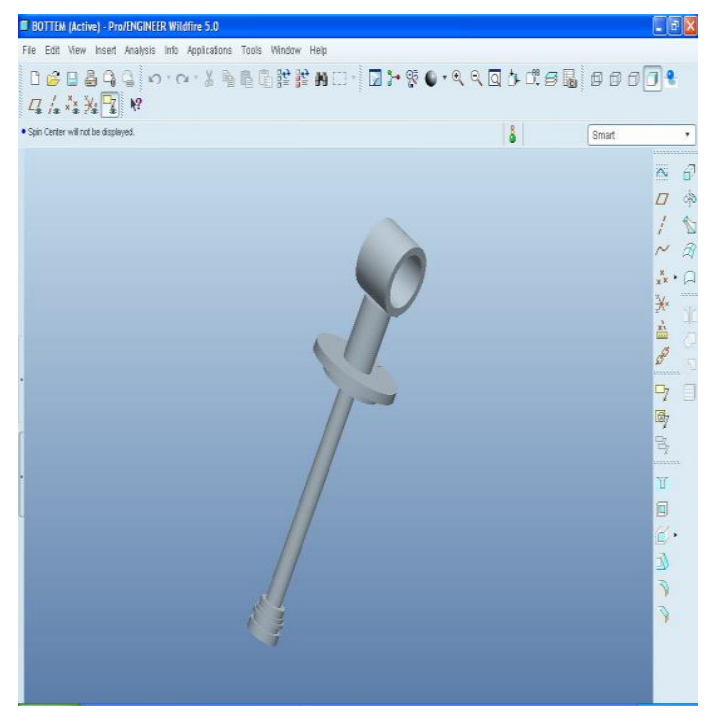

Fig 3.1:- Bottom part

\subsubsection{TOP PART}

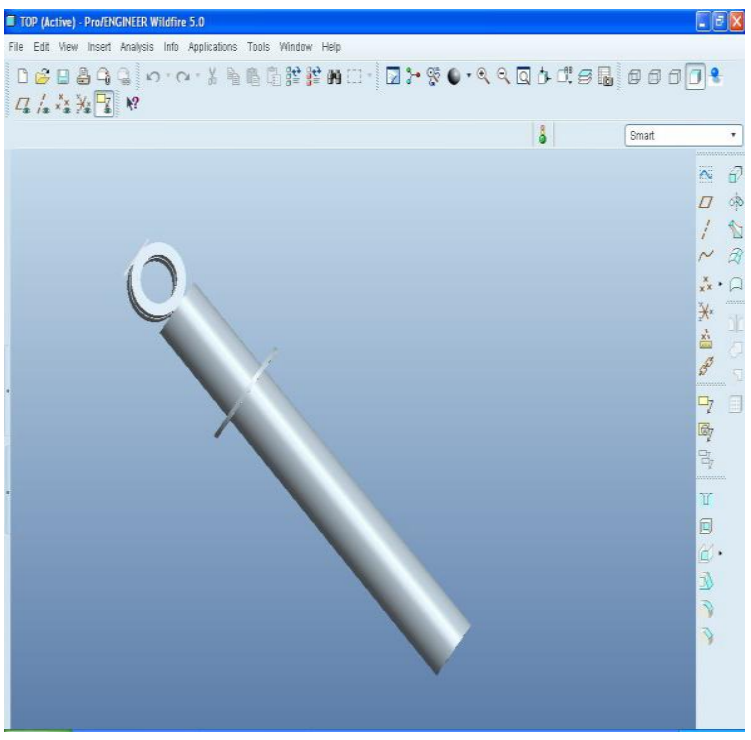

Fig 3.2:- Top part 


\subsubsection{HELICAL SPRING}

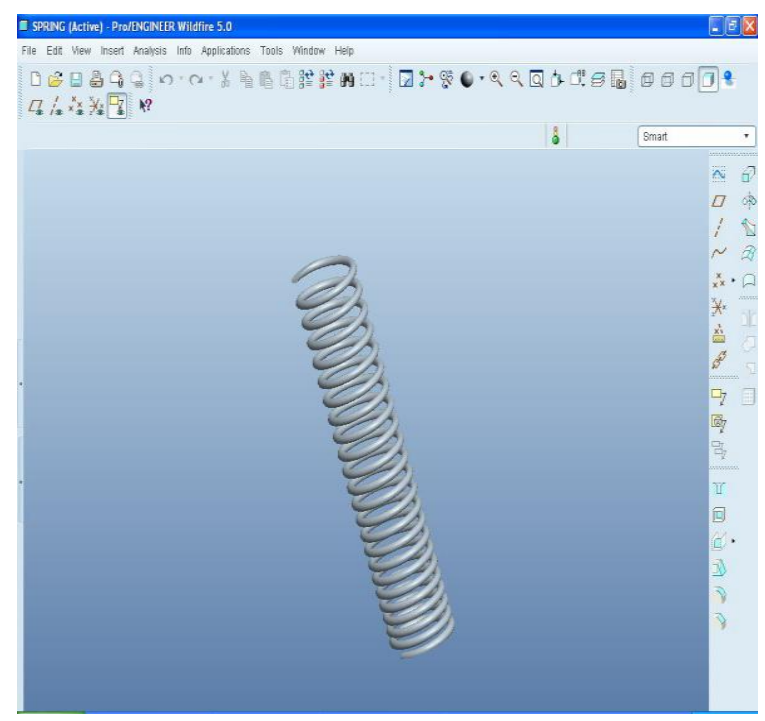

Fig 3.3:- Helical Spring

\subsection{TOTAL ASSEMBLY}

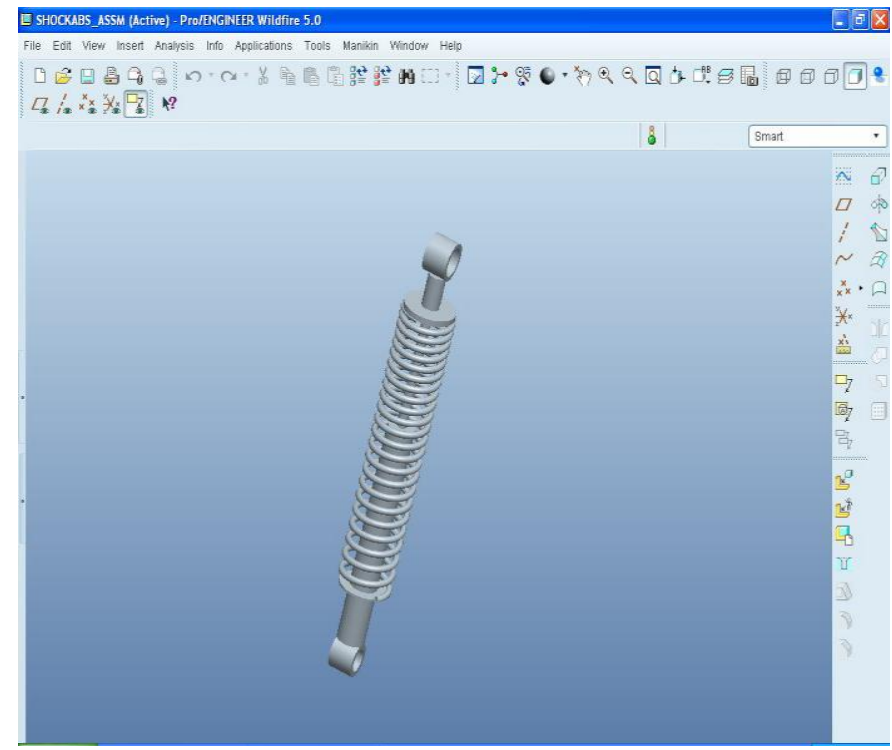

Fig 3.4:-Total Assembly

\subsection{D DRAWINGS OF SHOCK ABSORBER 3.4.1 BOTTOM}

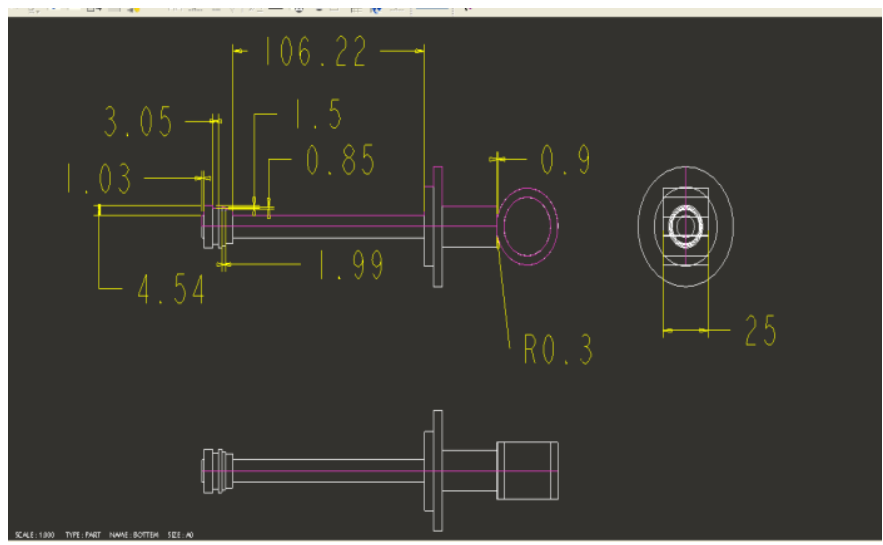

Fig 3.5:-Bottom part

\subsubsection{TOP PART}

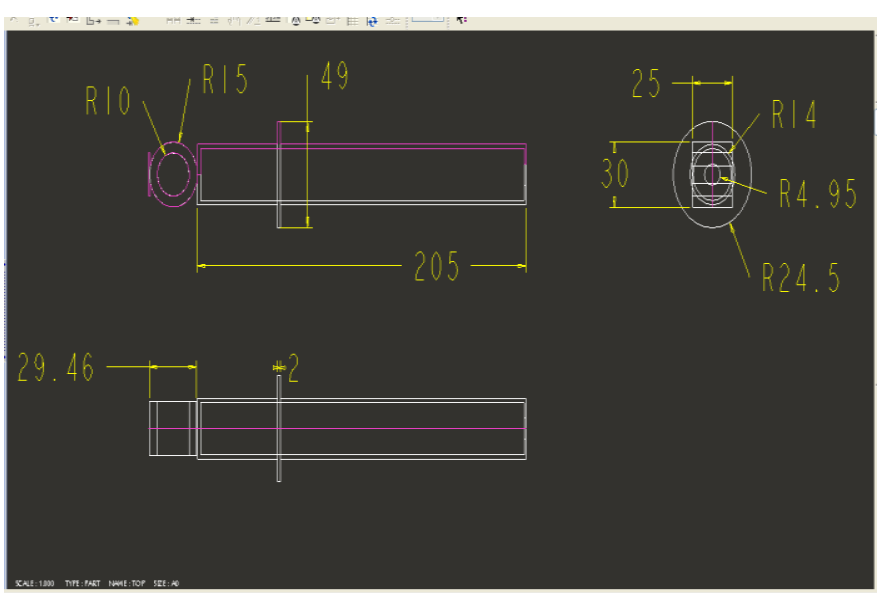

Fig 3.6:- Top part

\subsubsection{HELICAL SPRING PART}

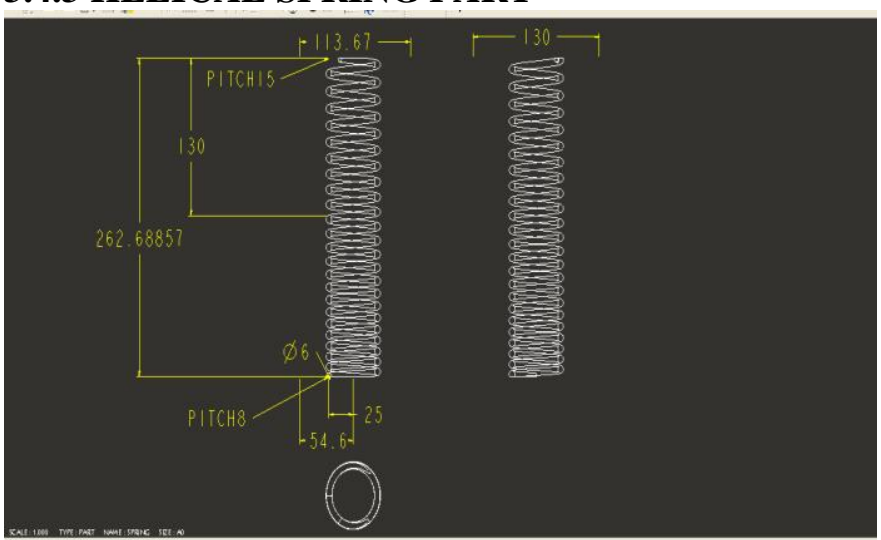

Fig 3.7:-Helical Spring part 


\subsection{ASSEMBLY}

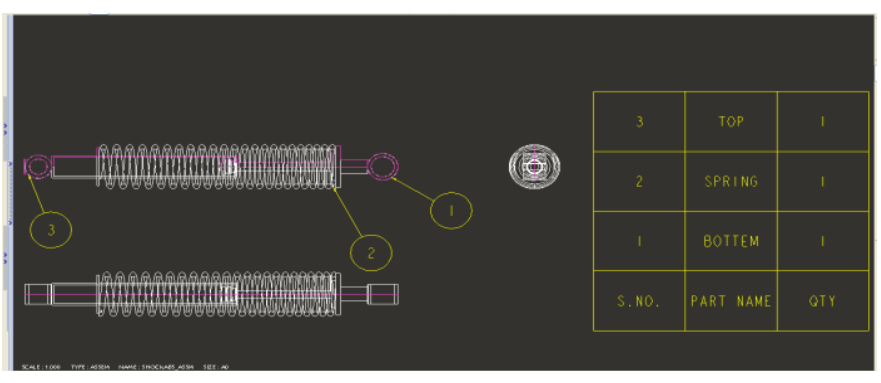

Fig 3.8:- Assembly

\subsection{MODIFIED SPRING OF SHOCK ABSORBER}

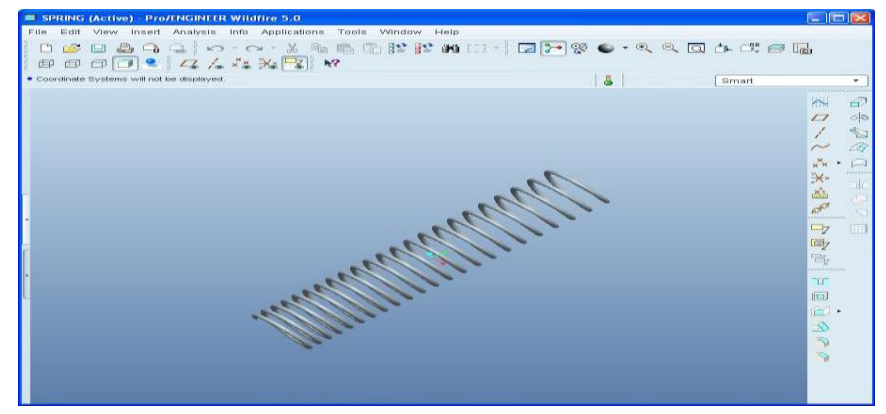

Fig 3.9:-Modified spring of shock absorber

\subsection{MODIFIED SPRING}

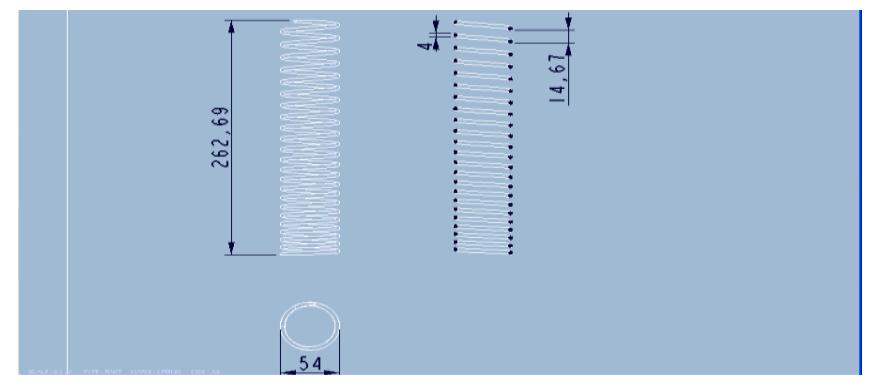

Fig 3.10:-Modified spring

\section{INTRODUCTION TO FEA}

Finite Element Analysis (FEA) was first developed in 1943 by R. Courant, who utilized the Ritz method of numerical analysis and minimization of variational calculus to obtain approximate solutions to vibration systems. Shortly thereafter, a paper published in 1956 by M. J. Turner, R. W. Clough, H. C. Martin, and L. J. Topp established a broader definition of numerical analysis. The paper centered on the "stiffness and deflection of complex structures". By the early 70's, FEA was limited to expensive mainframe computers generally owned by the aeronautics, automotive, defense, and nuclear industries. Since the rapid decline in the cost of computers and the phenomenal increase in computing power, FEA has been developed to an incredible precision. Present day supercomputers are now able to produce accurate results for all kinds of parameters. FEA consists of a computer model of a material or design that is stressed and analyzed for specific results. It is used in new product design, and existing product refinement. A company is able to verify a proposed design will be able to perform to the client's specifications prior to manufacturing or construction. Modifying an existing product or structure is utilized to qualify the product or structure for a new service condition. In case of structural failure, FEA may be used to help determine the design modifications to meet the new condition. There are generally two types of analysis that are used in industry: 2-D modeling, and 3-D modeling. While 2-D modeling conserves simplicity and allows the analysis to be run on a relatively normal computer, it tends to yield less accurate results. 3-D modeling, however, produces more accurate results while sacrificing the ability to run on all but the fastest computers effectively. Within each of these modeling schemes, the programmer can insert numerous algorithms (functions) which may make the system behave linearly or non-linearly. Linear systems are far less complex and generally do not take into account plastic deformation. Non-linear systems do account for plastic deformation, and many also are capable of testing a material all the way to fracture.

FEA uses a complex system of points called nodes which make a grid called a mesh. This mesh is programmed to contain the material and structural properties which define how the structure will react to certain loading conditions. Nodes are assigned at a certain density throughout the material depending on the anticipated stress levels of a particular area. Regions which will receive large amounts of stress usually have a higher node density than those which experience little or no stress. Points of interest may consist of: fracture point of previously tested material, fillets, corners, complex detail, and high stress areas. The mesh acts like a spider web in that from each node, there extends a mesh element to each of the adjacent nodes. This web of vectors is what carries the material properties to the object, creating many elements.

A wide range of objective functions (variables within the system) are available for minimization or maximization:

- Mass, volume, temperature

- Strain energy, stress strain

- Force, displacement, velocity, acceleration

- Synthetic (User defined)

There are multiple loading conditions which may be applied to a system. Some examples are shown:

- Point, pressure, thermal, gravity, and centrifugal static loads

- Thermal loads from solution of heat transfer analysis

- Enforced displacements

- Heat flux and convection 
- Point, pressure and gravity dynamic loads

Each FEA program may come with an element library, or one is constructed over time. Some sample elements are:

- Rod elements \& Beam elements

- Plate/Shell/Composite elements

- Shear panel

- Solid elements

- Spring elements

- Mass elements

- Rigid elements

- Viscous damping elements

Many FEA programs also are equipped with the capability to use multiple materials within the structure such as:

- Isotropic, identical throughout

- Orthotropic, identical at 90 degrees

- General anisotropic, different throughout

\subsection{Types of Engineering Analysis}

Structural analysis consists of linear and non-linear models. Linear models use simple parameters and assume that the material is not plastically deformed. Non-linear models consist of stressing the material past its elastic capabilities. The stresses in the material then vary with the amount of deformation as in. Vibrational analysis is used to test a material against random vibrations, shock, and impact. Each of these incidences may act on the natural Vibrational frequency of the material which, in turn, may cause resonance and subsequent failure. Fatigue analysis helps designers to predict the life of a material or structure by showing the effects of cyclic loading on the specimen. Such analysis can show the areas where crack propagation is most likely to occur. Failure due to fatigue may also show the damage tolerance of the material. Heat Transfer analysis models the conductivity or thermal fluid dynamics of the material or structure. This may consist of a steady-state or transient transfer. Steady-state transfer refers to constant thermo properties in the material that yield linear heat diffusion.

\subsection{Results of Finite Element Analysis}

FEA has become a solution to the task of predicting failure due to unknown stresses by showing problem areas in a material and allowing designers to see all of the theoretical stresses within. This method of product design and testing is far superior to the manufacturing costs which would accrue if each sample was actually built and tested. In practice, a finite element analysis usually consists of three principal steps.

\subsubsection{Preprocessing:}

The user constructs a model of the part to be analyzed in which the geometry is divided into a number of discrete sub regions, or elements," connected at discrete points called nodes." Certain of these nodes will have fixed displacements, and others will have prescribed loads. These models can be extremely time consuming to prepare, and commercial codes vie with one another to have the most user-friendly graphical "preprocessor" to assist in this rather tedious chore. Some of these preprocessors can overlay a mesh on a preexisting CAD file, so that finite element analysis can be done conveniently as part of the computerized drafting-and-design process.

\subsubsection{Analysis:}

The dataset prepared by the preprocessor is used as input to the finite element code itself, which constructs and solves a system of linear or nonlinear algebraic equations.

$$
\text { Kijuj }=\mathrm{fi}
$$

where $\mathrm{u}$ and $\mathrm{f}$ are the displacements and externally applied forces at the nodal points; The formation of the $\mathrm{K}$ matrix is dependent on the type of problem being attacked, and this module will outline the approach for truss and linear elastic stress analyses; Commercial codes may have very large element libraries, with elements appropriate to a wide range of problem types. One of FEA's principal advantages is that many problem types can be addressed with the same code, merely by specifying the appropriate element types from the library.

\subsubsection{Post-processing:}

In the earlier days of finite element analysis, the user would pore through reams of numbers generated by the code, listing displacements and stresses at discrete positions within the model. It is easy to miss important trends and hot spots this way, and modern codes use graphical displays to assist in visualizing the results. Typical postprocessor display overlays colored contours representing stress levels on the model, showing a full field picture similar to that of photo elastic or moiré experimental results.

\section{INTRODUCTION TO ANSYS}

ANSYS is general-purpose finite element analysis (FEA) software package. Finite Element Analysis is a numerical method of deconstructing a complex system into very small pieces (of user-designated size) called elements. The software Implements equations that govern the behavior of these elements and solves them all; creating a comprehensive explanation of how the system acts as a whole. These results then can be presented in tabulated, or graphical forms. This type of analysis is typically used for the design and optimization of a system far too complex to analyze by hand. Systems that may fit into this category are too complex due to their geometry, scale, or governing equations.

ANSYS is the standard FEA teaching tool within the Mechanical Engineering Department at many colleges. ANSYS is also used in Civil and Electrical Engineering, as 
well as the Physics and Chemistry departments. ANSYS provides a cost-effective way to explore the performance of products or processes in a virtual environment. This type of product development is termed virtual prototyping. With virtual prototyping techniques, users can iterate various scenarios to optimize the product long before the manufacturing is started. This enables a reduction in the level of risk, and in the cost of ineffective designs. The multifaceted nature of ANSYS also provides a means to ensure that users are able to see the effect of a design on the whole behavior of the product, be it electromagnetic, thermal, mechanical etc.

\subsection{Generic Steps to Solving any Problem in ANSYS:}

Like solving any problem analytically, you need to define (1) your solution domain, (2) the physical model, (3) boundary conditions and (4) the physical properties. You then solve the problem and present the results. In numerical methods, the main difference is an extra step called mesh generation. This is the step that divides the complex model into small elements that become solvable in an otherwise too complex situation. Below describes the processes in terminology slightly more attune to the software.

\subsubsection{Build Geometry}

Construct a two or three dimensional representation of the object to be modeled and tested using the work plane coordinates system within ANSYS.

\subsubsection{Define Material Properties}

Now that the part exists, define a library of the necessary materials that compose the object (or project) being modeled. This includes thermal and mechanical properties.

\subsubsection{Generate Mesh}

At this point ANSYS understands the makeup of the part. Now define how the Modeled system should be broken down into finite pieces.

\subsubsection{Apply Loads}

Once the system is fully designed, the last task is to burden the system with constraints, such as physical loadings or boundary conditions.

\subsubsection{Obtain Solution}

This is actually a step, because ANSYS needs to understand within what state (steady state, transient... etc.) the problem must be solved.

\subsubsection{Present the Results}

After the solution has been obtained, there are many ways to present ANSYS' results, choose from many options such as tables, graphs, and contour plots.

\subsection{Specific Capabilities of ANSYS:}

\subsubsection{Structural}

Structural analysis is probably the most common application of the finite element method as it implies bridges and buildings, naval, aeronautical, and mechanical structures such as ship hulls, aircraft bodies, and machine housings, as well as mechanical components such as pistons, machine parts, and tools.

\subsubsection{Static Analysis}

Used to determine displacements, stresses, etc. under static loading conditions. ANSYS can compute both linear and nonlinear static analyses. Nonlinearities can include plasticity, stress stiffening, large deflection, large strain, hyper elasticity, contact surfaces, and creep.

\subsubsection{Transient Dynamic Analysis}

Used to determine the response of a structure to arbitrarily time-varying loads. All nonlinearities mentioned under Static Analysis above are allowed.

\subsubsection{Buckling Analysis}

Used to calculate the buckling loads and determine the buckling mode shape. Both linear (eigenvalue) buckling and nonlinear buckling analyses are possible. In addition to the above analysis types, several special-purpose features are available such as Fracture mechanics, Composite material analysis, Fatigue, and both p-Method and Beam analyses.

\subsubsection{Thermal Analysis}

ANSYS is capable of both steady state and transient analysis of any solid with thermal boundary conditions. Steady-state thermal analyses calculate the effects of steady thermal loads on a system or component. Users often perform a steady-state analysis before doing a transient thermal analysis, to help establish initial conditions. A steady-state analysis also can be The last step of a transient thermal analysis; performed after all transient effects have diminished. ANSYS can be used to determine temperatures, thermal gradients, heat flow rates, and heat fluxes in an object that are caused by thermal loads that do not vary over time. Such loads include the following:

- Convection

- Radiation

- Heat flow rates

- Heat fluxes (heat flow per unit area)

- Heat generation rates (heat flow per unit volume)

- Constant temperature boundaries

A steady-state thermal analysis may be either linear, with constant material properties; or nonlinear, with material properties that depend on temperature. The thermal properties 
of most material vary with temperature. This temperature dependency being appreciable, the analysis becomes nonlinear. Radiation boundary conditions also make the analysis nonlinear. Transient calculations are time dependent and ANSYS can both solve distributions as well as create video for time incremental displays of models.

\subsubsection{Acoustics / Vibration Analysis}

ANSYS is capable of modeling and analyzing vibrating systems in order to that vibrate in order to analyze. Acoustics is the study of the generation, propagation, absorption, and reflection of pressure waves in a fluid medium. Applications for acoustics include the following:

Sonar - the acoustic counterpart of radar

Design of concert halls, where an even distribution of sound pressure is desired

Noise minimization in machine shops

Noise cancellation in automobiles

Underwater acoustics

Design of speakers, speaker housings, acoustic filters, mufflers, and many other similar devices.

Geophysical exploration

Within ANSYS, an acoustic analysis usually involves modeling a fluid medium and the surrounding structure. Characteristics in question include pressure distribution in the fluid at different frequencies, pressure gradient, and particle velocity, the sound pressure level, as well as, scattering, diffraction, transmission, radiation, attenuation, and dispersion of acoustic waves. A coupled acoustic analysis takes the fluidstructure interaction into account. An uncoupled acoustic analysis models only the fluid and ignores any fluid-structure interaction. The ANSYS program assumes that the fluid is compressible, but allows only relatively small pressure changes with respect to the mean pressure. Also, the fluid is assumed to be non-flowing and inviscid (that is, viscosity causes no dissipative effects). Uniform mean density and mean pressure are assumed, with the pressure solution being the deviation from the mean pressure, not the absolute pressure.

\subsubsection{Coupled Fields Analysis}

A coupled-field analysis is an analysis that takes into account the interaction (coupling) between two or more disciplines (fields) of engineering. A piezoelectric analysis, for example, handles the interaction between the structural and electric fields: it solves for the voltage distribution due to applied displacements, or vice versa. Other examples of coupled-field analysis are thermal-stress analysis, thermal-electric analysis, and fluid-structure analysis. Some of the applications in which coupled-field analysis may be required are pressure vessels (thermal-stress analysis), fluid flow constrictions (fluidstructure analysis), induction heating (magnetic-thermal analysis), ultrasonic transducers (piezoelectric analysis), magnetic forming (magneto-structural analysis), and micro electro mechanical systems (MEMS).

\subsubsection{Modal Analysis}

A modal analysis is typically used to determine the vibration characteristics (natural frequencies and mode shapes) of a structure or a machine component while it is being designed. It can also serve as a starting point for another, more detailed, dynamic analysis, such as a harmonic response or full transient dynamic analysis. Modal analyses, while being one of the most basic dynamic analysis types available in ANSYS, can also be more computationally time consuming than a typical static analysis. A reduced solver, utilizing automatically or manually selected master degrees of freedom is used to drastically reduce the problem size and solution time.

\subsubsection{Harmonic Analysis}

Used extensively by companies who produce rotating machinery, ANSYS Harmonic analysis is used to predict the sustained dynamic behavior of structures to consistent cyclic loading. Examples of rotating machines which produced or are Subjected to harmonic loading are:

- Gas Turbines for Aircraft and Power Generation

- Steam Turbines

- Wind Turbine

- Water Turbines

- Turbo pumps

- Internal Combustion engines

- Electric motors and generators

- Gas and fluid pumps

- Disc drives

A harmonic analysis can be used to verify whether or not a machine design will successfully overcome resonance, fatigue, and other harmful effects of forced vibrations.

\section{PRESENT DESIGN}

\subsection{Structural Analysis for bike weight (125kgs) using} Spring Steel as spring material

\section{Case 1: Load 125kgs}

Element Type Solid 20 node 95

Material: Spring Steel

Material Properties: Young's Modulus (EX): 210000N/mm2

Poisson's Ratio (PRXY): 0.29

Density: $0.000007850 \mathrm{~kg} / \mathrm{mm} 3$ 


\subsubsection{Imported Model from Pro/Engineer}

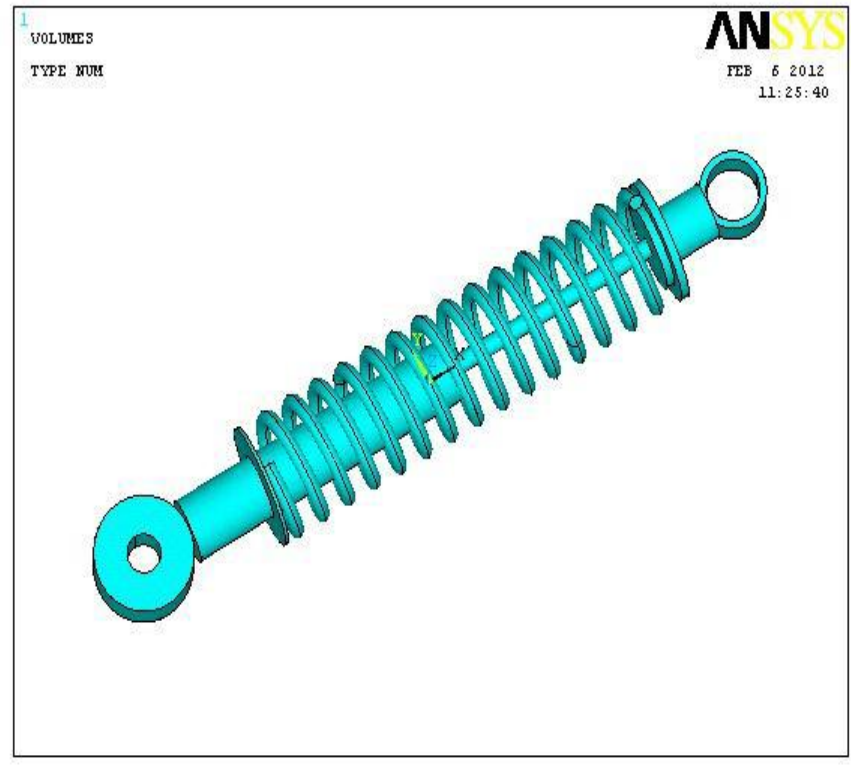

Fig 6.1:- Imported model from Pro/Engineer

\subsubsection{Tetra Meshed Model}

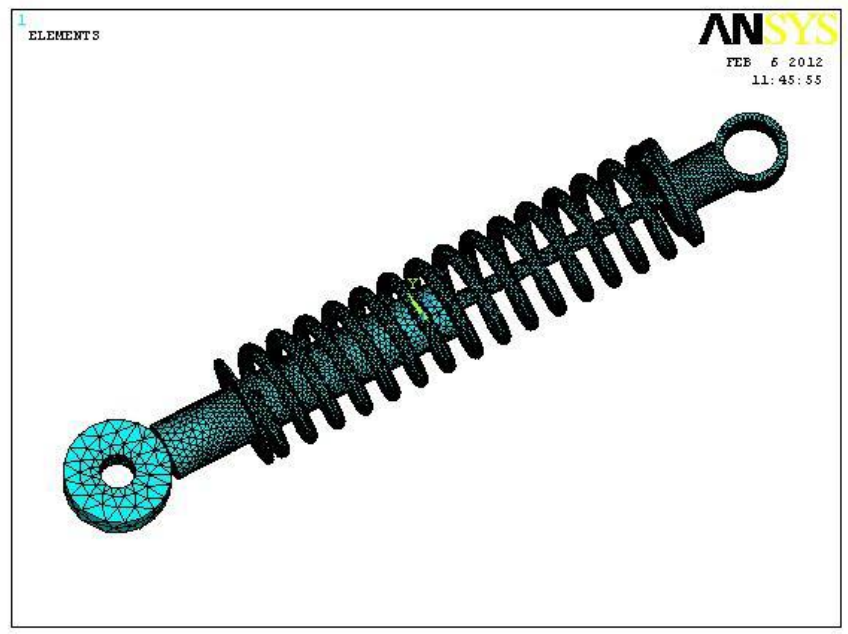

Fig 6.2:- Tetra Meshed model

Loads

Pressure $-0.0078 \mathrm{~N} / \mathrm{mm} 2$

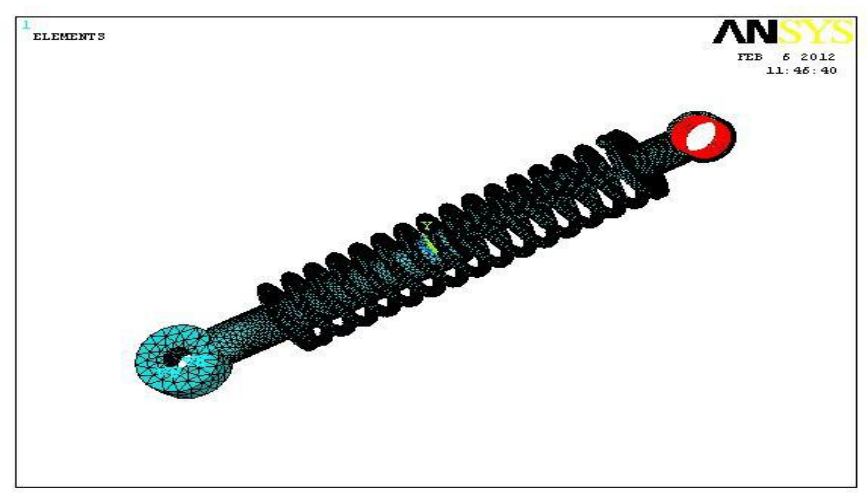

Fig 6.3:- Load applied on tetra mesh model

\section{RESULTS AFTER APPLIENG LOADS:}

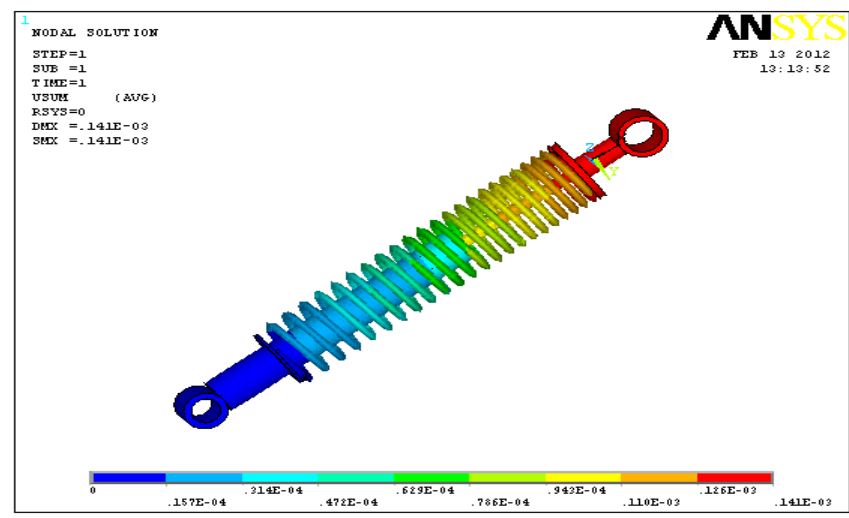

Fig 6.4:- Displacement Vector Sum

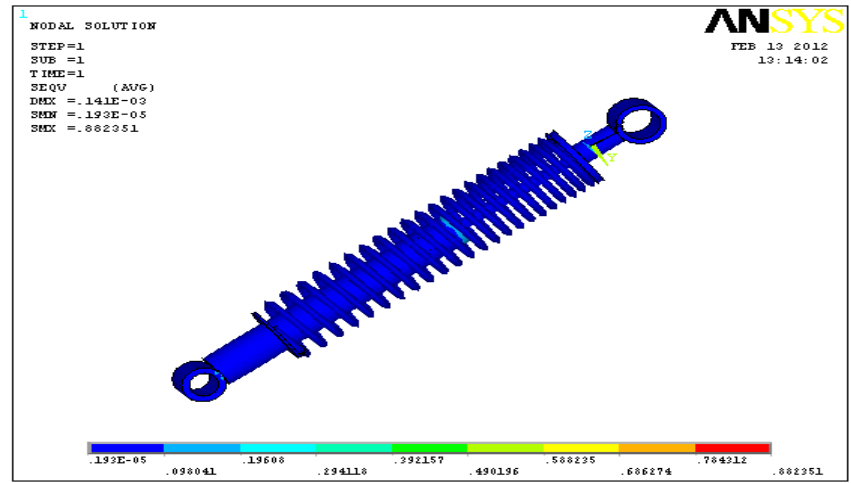

Fig 6.5:- Von misses stress

6.2 Structural Analysis for bike weight (125kgs) using Beryllium Copper as spring material

Case 2: Load 125kg

Element Type: solid 20 node 95

Material: Beryllium Copper

Material Properties: Young's Modulus (EX) : 280000N/mm2 
Poisson's Ratio (PRXY): 0.285

Density: $0.000001850 \mathrm{~kg} / \mathrm{mm} 3$

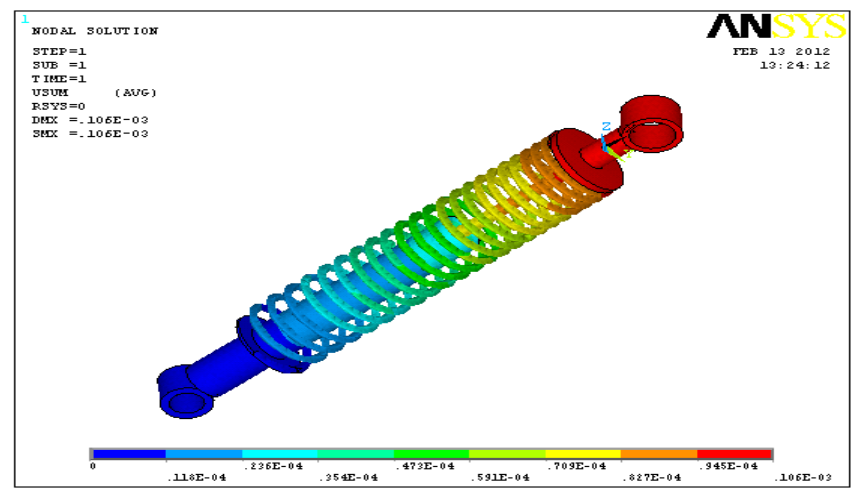

Fig 6.6:- Displacement vector sum

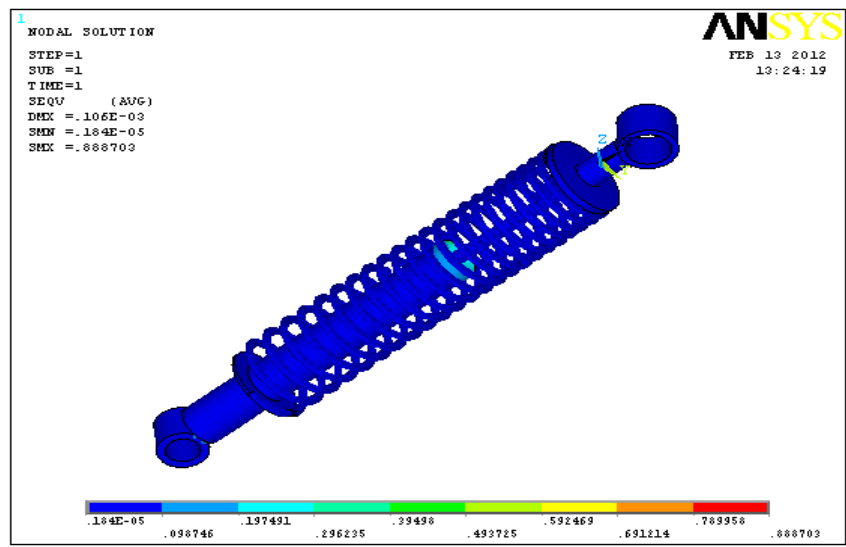

Fig 6.7:-Von misses stress

\subsection{Structural Analysis for one person and bike} weight (200kgs) using Spring Steel as spring material Case 3: Load 200kg

Element Type Solid 20 node 95

Material: Spring Steel

Material Properties: Young's Modulus (EX) : 210000N/mm2

Poisson's Ratio (PRXY) : 0.29

Density: $0.000007850 \mathrm{~kg} / \mathrm{mm} 3$

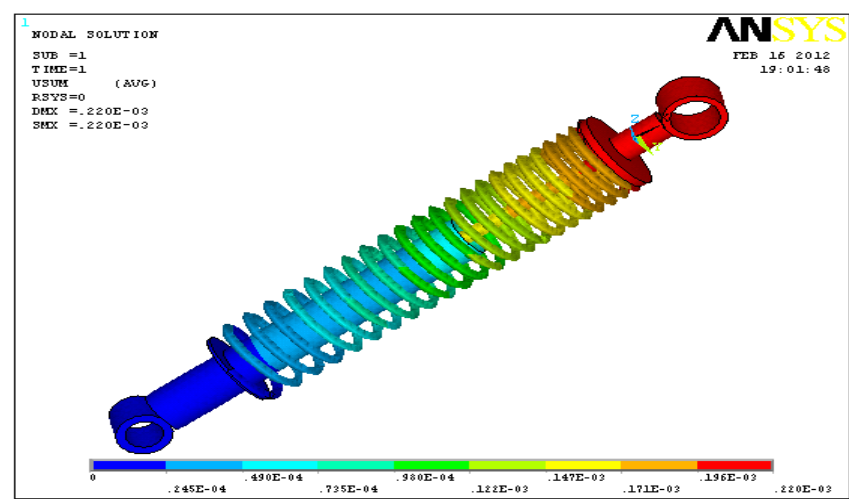

Fig 6.8:-Displacement vector sum

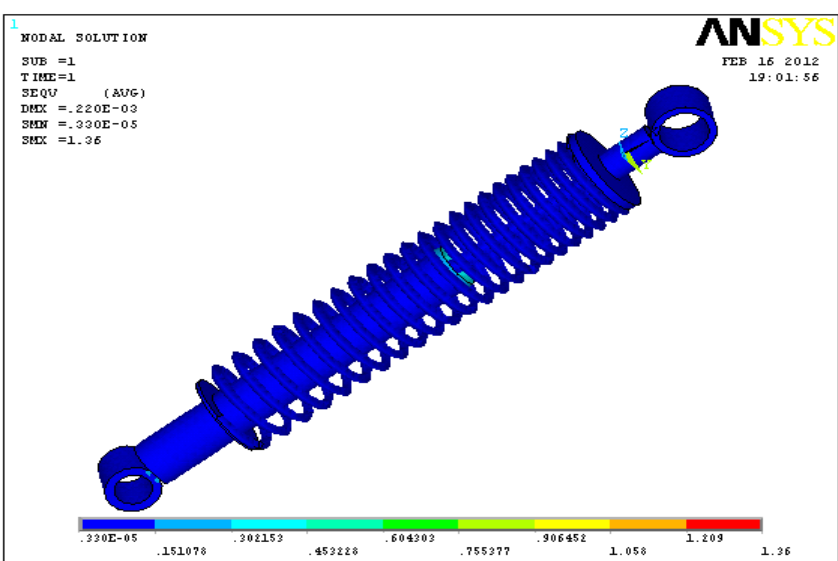

Fig 6.9:-Van misses stress

6.3 Structural Analysis for one person and bike weight $(200 \mathrm{kgs})$ using

\section{Beryllium Copper as spring material}

\section{Case 4: Load 200kgs}

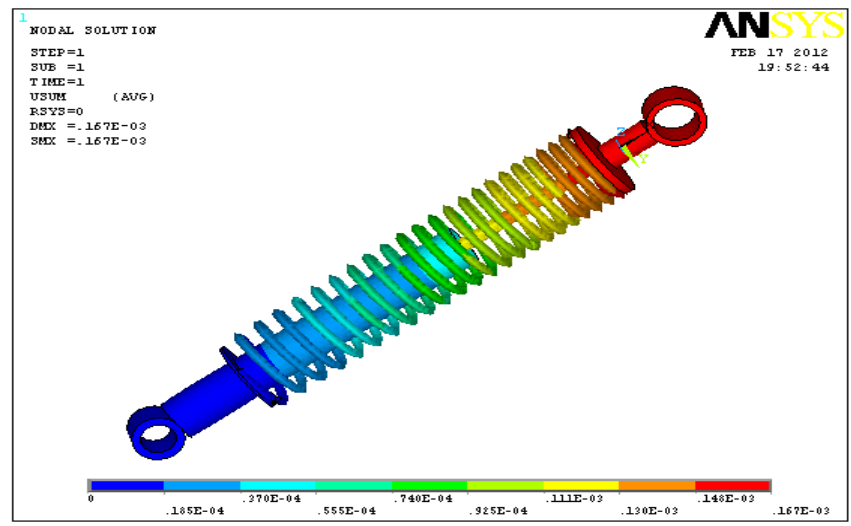

Fig 6.10:-Displacement vector sum 


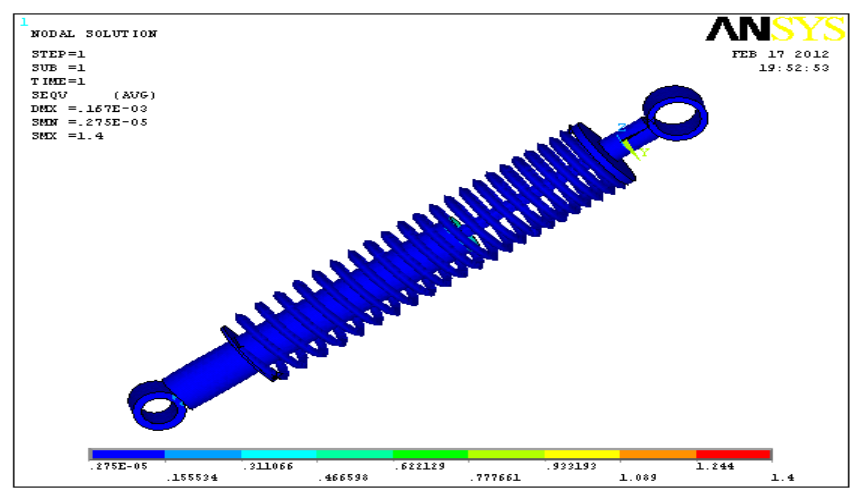

Fig 6.11:- Von misses stress

\section{NEW MODIFIED DESIGN}

7.1 Structural Analysis for bike weight (125kgs) using Spring Steel as spring material

Case 1: Load 125kgs

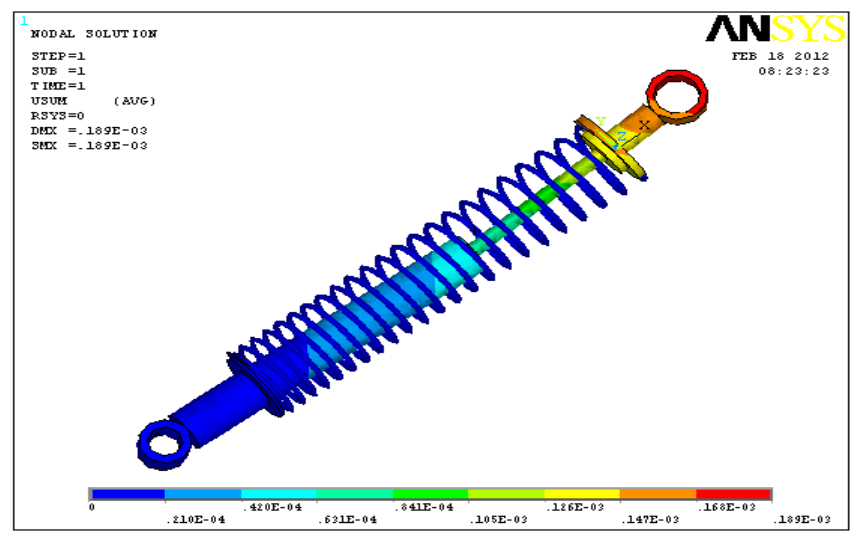

Fig 7.1:-Displacement vector sum

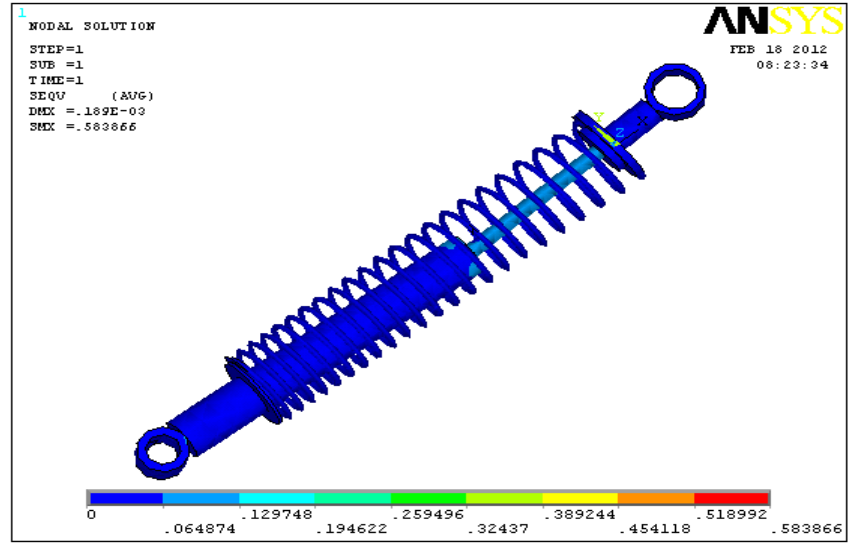

Fig 7.2:-Von misses stress
7.2 Structural Analysis for bike weight (125kgs) using Beryllium Copper as spring material

Case 2: Load 125kgs

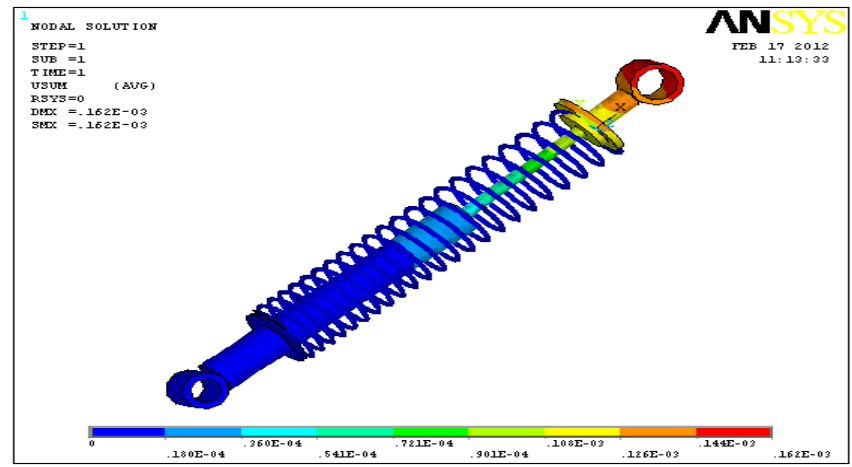

Fig 7.3:- Displacement vector sum

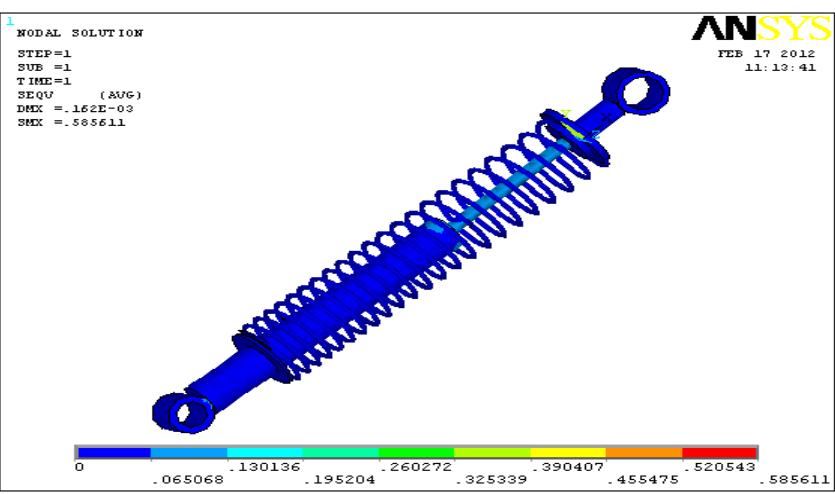

Fig 7.4:- Von misses stress

7.3 Structural Analysis for one person and bike weight(200kgs) using Spring Steel as spring material Case 3: Load 200kgs

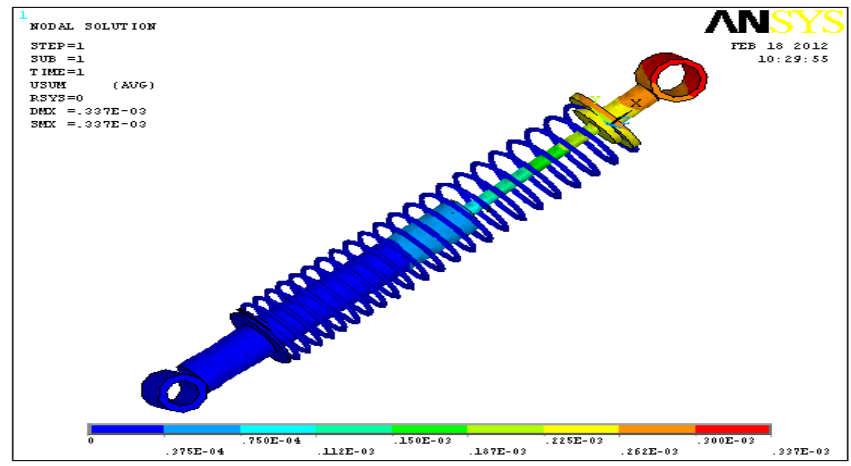

Fig 7.5:- Displacement vector sum 


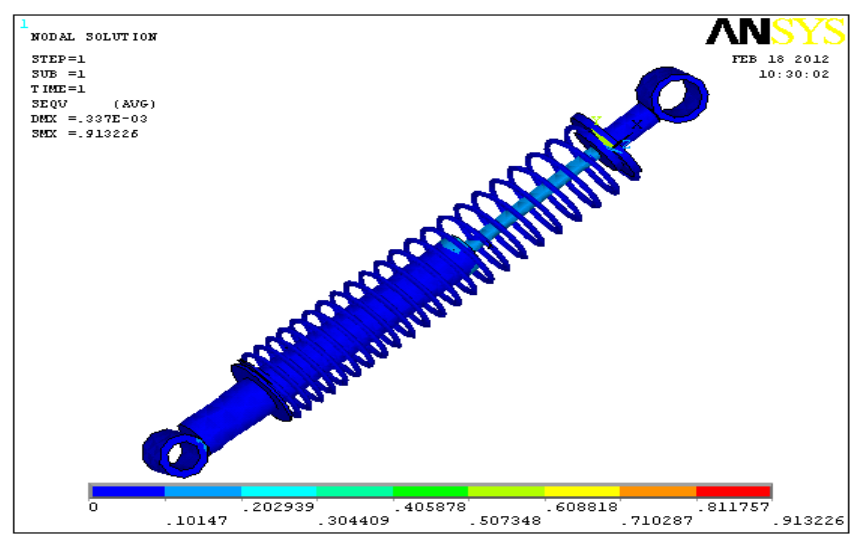

Fig 7.6:-Von Misses Stress

\subsection{Structural Analysis for one person and bike} weight (200kgs) using Beryllium Copper as spring material

\section{Case 4: Load 200kgs}

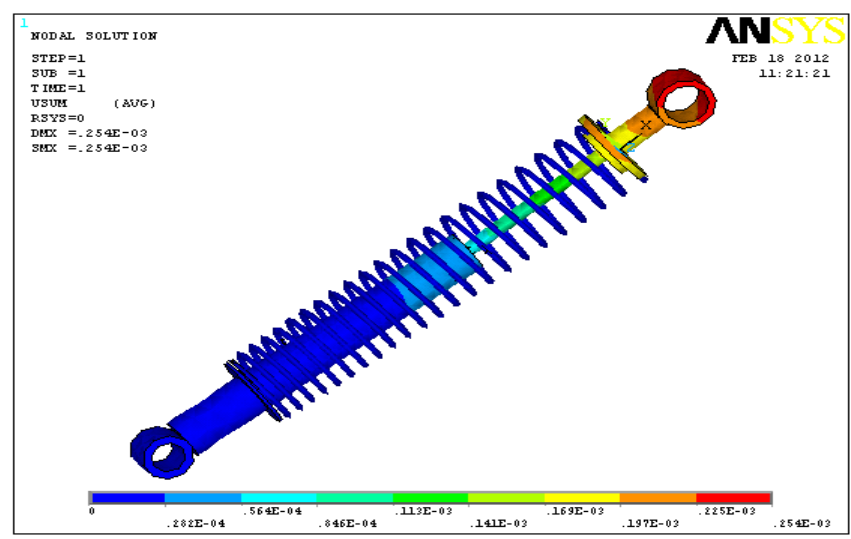

Fig 7.7:- Displacement vector sum

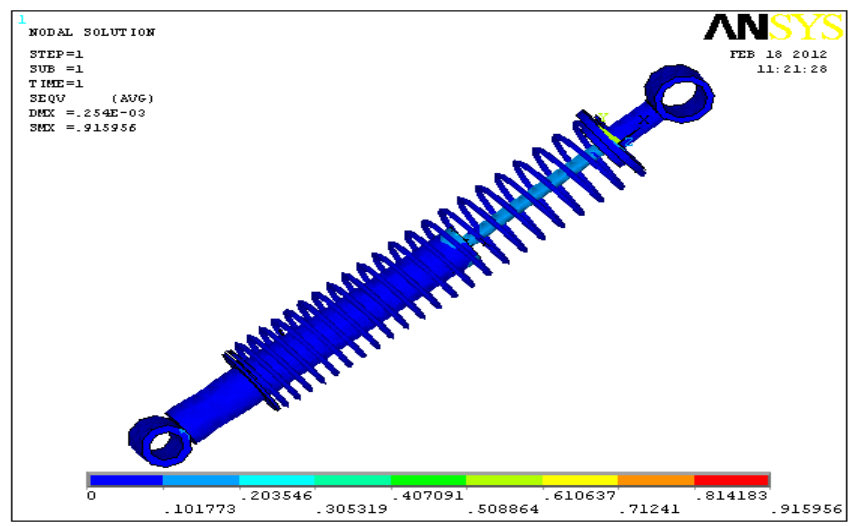

Fig 7.8:- Von misses stress

\section{RESULTS TABLE:}

\subsection{PRESENT DESIGN}

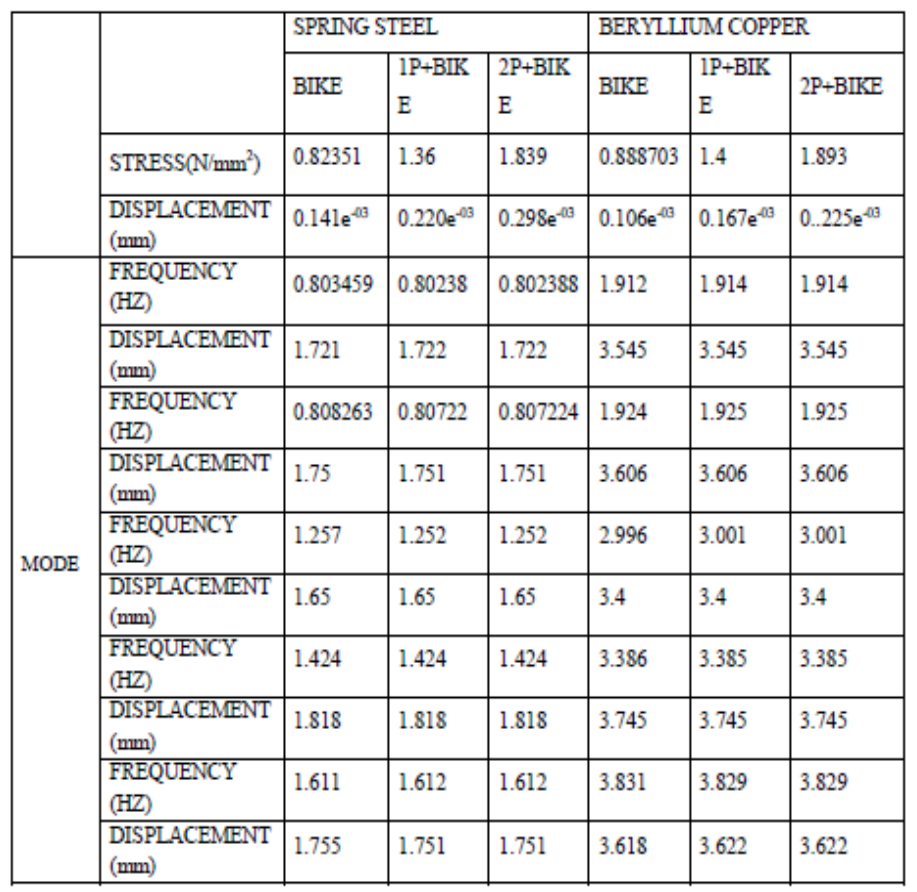

\subsection{MODIFIED DESIGN}

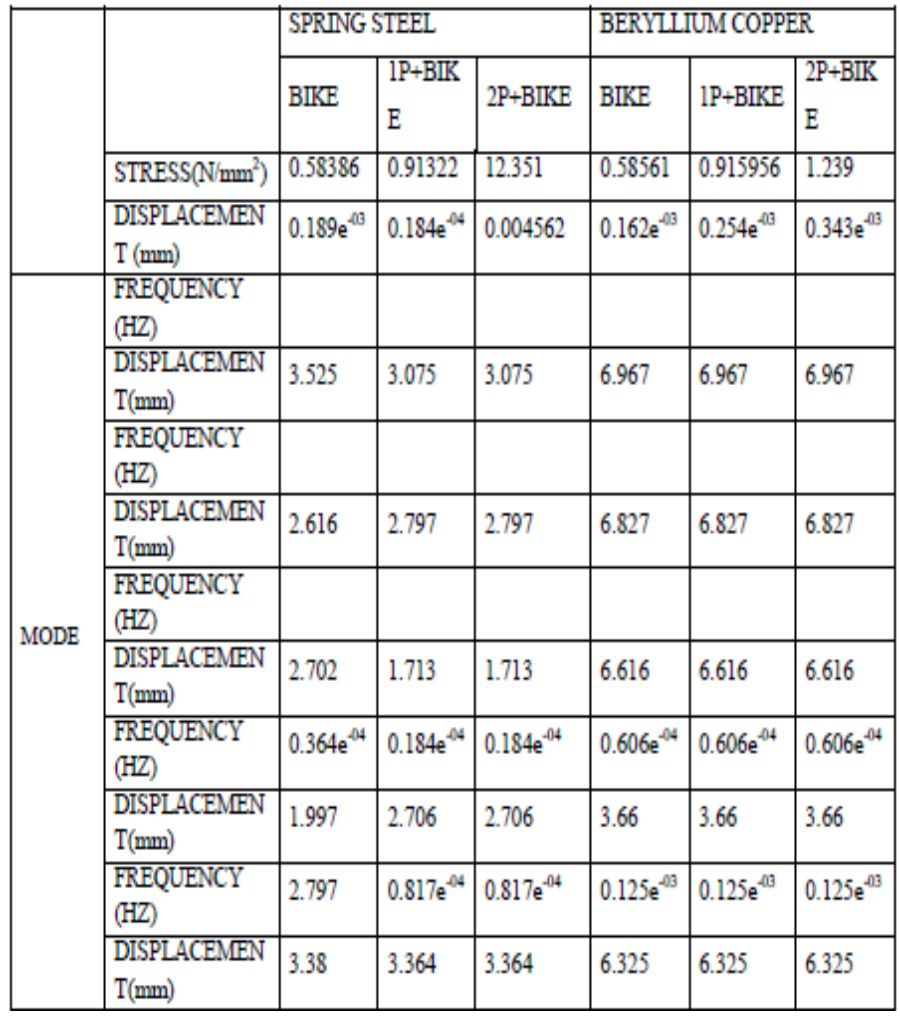




\section{CONCLUSIONS}

[1]. In this project we have designed a shock absorber used in a $150 \mathrm{cc}$ bike. We have modeled the shock absorber by using 3D parametric software Pro/Engineer.

[2]. To validate the strength of our design, we have done structural analysis and modal analysis on the shock absorber. We have done analysis by varying spring material Spring Steel and Beryllium Copper.

[3]. By observing the analysis results, the analyzed stress values are less than their respective yield stress values. So our design is safe.

[4]. By comparing the results for both materials, the stress value is less for Spring Steel than Beryllium Copper.

[5]. Also the shock absorber design is modified by reducing the diameter of spring by $2 \mathrm{~mm}$ and structural, modal analysis is done on the shock absorber. By reducing the diameter, the weight of the spring reduces. By comparing the results for both materials, the stress value is less for Spring Steel than Beryllium Copper.

[6]. By comparing the results for present design and modified design, the stress and displacement values are less for modified design.

[7]. So we can conclude that as per our analysis using material spring steel for spring is best and also our modified design is safe.

\section{REFERENCES}

[1]. Machine design by R.S.KURMI

[2]. PSG,2008."DESIGN DATA," kalaikathir achachgam publishers, COIMBATORE, INDIA

[3]. Automobile Engineering by R.B.Gupta

[4]. Automobile Engineering by G.B.S. Narang.

[5]. Automobile Servicing and Maintenance by K.Ashrif Ali

[6]. Automotive Maintenance and Trouble Shooting by Ernest Venk,\& Edward D. Spicer 